\title{
MEJORAMIENTO DE LA VIDA ÚTIL (Shelf life) DE LA BEBIDA TRADICIONAL PERUANA EMOLIENTE
}

\author{
IMPROVEMENT OF THE SHELF LIFE OF THE TRADITIONAL \\ PERUVIAN EMOLLIENT DRINK
}

\author{
${ }^{1}$ Enrique Alfonso De Florio Ramírez, ${ }^{2}$ Liliana del Carmen Lanchipa Bergamini, ${ }^{3}$ Mario Alberto Matos Peñas
}

\begin{abstract}
RESUMEN
En la presente investigación se estudió el mejoramiento de la vida útil del emoliente peruano, cuya materia prima estuvo constituida por los siguientes ingredientes: cola de caballo (Equisetum giganteun) (14,9\%), boldo (Peumus boldus) $(0,6 \%)$, flor de arena (Tiquilia paromychiodes) $(1,1 \%)$, grama (Elymus repens) $(0,4 \%)$, uña de gato (Uncaria tomentosa) (6,5\%), linaza (Linum usitatissimum) $(29,7 \%)$, cebada tostada (Hordemu vulgare) $(46,8 \%)$. Estos fueron sometidos a cocción por espacio de 3 horas, seguidamente se adicionó el edulcorante natural estevia (Stevia rebaudiana bertoni) y se bajó el $\mathrm{pH}$ a 4,2. Más adelante, se envasó en botellas de $300 \mathrm{ml}$ y se expuso a una pasteurización de $90^{\circ}$ centígrados por un periodo de 15 minutos; al término del cual, las muestras fueron colocadas en un ambiente sin luz y a temperatura constante de $22^{\circ}$ centígrados. Con el objetivo de determinar la vida útil de este emoliente se utilizó la evaluación sensorial mediante un panel semi entrenado, que estuvo integrado por 11 estudiantes del quinto año de la ESIA-UNBG; durante 10 sesiones (15 semanas) se registraron los datos correspondientes y aplicando métodos estadísticos, se estimó la vida útil en 114 días.
\end{abstract}

Palabras clave: Bebidas tradicionales, emoliente peruano, vida útil.

\begin{abstract}
In the present investigation the improvement of the useful life of the Peruvian emollient was studied, whose raw material was constituted by the following ingredients: horsetail (Equisetum giganteum) (14.9\%), boldo (Peumus boldus) $(0,6 \%)$, sand flower (Tiquilia paromychiodes) $(1,1 \%)$, grass (Elymus repens) $(0.4 \%)$, cat's claw (Uncaria tomentosa) $(6,5 \%)$, flaxseed (Linum usitatissimum) (29,7\%), toasted barley (Hordemu vulgare) (46,8\%). These ingredients were subjected to cooking for 3 hours, then a natural stevia sweetener (Stevia rebaudiana bertoni) was added and the $\mathrm{pH}$ was lowered to 4,2; packed in $300 \mathrm{ml}$ bottles taking a $90^{\circ} \mathrm{C}$ pasteurization for 15 minutes. The samples were placed in an environment without light and at a constant temperature of $22^{\circ} \mathrm{Celsius}$. To determine the useful life, the sensory evaluation was used by a semi-trained panel formed by 11 students of the fifth year of the ESIA-UNBG during 10 sessions (15 weeks) and with this data, statistical methods were used to estimate the useful life in 114 days.
\end{abstract}

Keywords: Traditional drinks, emollient Peruvian, shelflife.

\section{INTRODUCCIÓN}

La Real Academia Española, según refiere Acosta (2011), define al emoliente peruano como una "bebida medicinal que resulta del cocimiento ligero de una o varias hierbas y otros ingredientes en agua"; la cual queda impregnada de sustancias solubles que aportan efectos beneficiosos para la salud.

Aún no puede determinarse el origen exacto de esta preparación; sin embargo, se sabe que en España la cebada ha sido considerada como medicinal desde mucho antes de la llegada de los españoles a América. Así mismo, los habitantes del Tahuantinsuyo solían tratar sus males orgánicos, espirituales o emocionales con infusiones calientes y zumos extraídos de raíces, tallos, flores y frutos. Con la Conquista, las costumbres $\mathrm{y}$ creencias del pueblo andino fueron reprimidas o transformadas; no obstante, el consumo del emoliente peruano continuó practicándose a lo largo de toda la época colonial y su consumo se ha incrementado en

\footnotetext{
'Departamento de Ingeniería de Industrias Alimentarias. Facultad de Ciencias Agropecuarias. Universidad Nacional Jorge Basadre Grohmann. Tacna- Perú. E-mail: deflorioenrique@gmail.com

${ }^{2}$ Departamento de Ingeniería de Industrias Alimentarias. Facultad de Ciencias Agropecuarias. Universidad Nacional Jorge Basadre Grohmann. Tacna- Perú. E-mail: lilianne10000@hotmail.com

Departamento de Matemáticas. Facultad de Ciencias. Universidad Nacional Jorge Basadre Grohmann.Tacna- Perú. E-mail: matosmp@gmail.com
} 
nuestros días (Emoliente etnobotánica, 2010).

El emoliente está hecho a base de hierbas consideradas medicinales, puesto que poseen propiedades beneficiosas para la salud. Según sea el país, se ha denominado a esta bebida con diferentes nombres y sus usos varían desde una medicina alternativa que promueve la curación, hasta una simple sensación de alivio frente a los síntomas provocados por diversos males. (Vida OK, 2013).

Usualmente, se señala un tiempo de vida de 48 horas para esta preparación; aunque se han encontrado patentes de emoliente pasteurizado, sin indicación alguna sobre temperaturas, tiempos de pasteurizado, ni vida útil del mismo (Datos Perú, 2000).

El denominador común de todo emoliente es el empleo de materia prima como la cebada y la linaza; así también, se incorporan otros productos cultivados en nuestro país. Existen muchas variantes en cuanto a estos ingredientes y el efecto refrescante/medicinal que ofrece las diversas combinaciones que el cliente solicita (El cocinero peruano, 2018). La elección de la stevia (Stevia reberdiana Bertoni) como endulzante se debe a que resulta una buena alternativa contra la diabetes, ya que no afecta negativamente a los niveles de azúcar en la sangre (ecoticias.com, 2015).

Aunque en la actualidad el emoliente peruano se venda como un producto ambulatorio, existen iniciativas para industrializarlo y difundirlo en el mercado latinoamericano, a fin de promover el conocimiento de sus propiedades en beneficio de la salud y el bienestar de los ciudadanos, además de ser una bebida naturalmente refrescante (Montes, 2012).

Labuza (1999) — citado en los informes de la Biblioteca Central de la Universidad Nacional de Santa (2011) - indica que desde el punto de vista de la seguridad y del aspecto organoléptico, el periodo que retendrá un nivel aceptable de la calidad alimenticia de un producto, esto es, la vida útil de un alimento depende de cuatro factores principales: conocer la formulación, el procesado, el empacado y las condiciones de almacenamiento.

Para evaluar el tiempo de vida útil será necesario definir indicadores de calidad que varían en función del tiempo, entre estos indicadores está el panel sensorial. (Posada, 2011).

El incremento de la vida útil del emoliente es de vital importancia para mejorar la comercialización e industrialización y así exportarlo a países latinoamericanos vecinos, donde es reconocido por sus propiedades refrescante y nutrasenicas

Dentro de este marco, la presente investigación tiene como objetivo principal el mejoramiento de la vida útil o durabilidad del emoliente peruano, aplicando para ello la pasteurización como método.

\section{MATERIALES Y MÉTODOS}

La presente investigación es de tipo Aplicada, nivel Descriptivo y diseño Experimental. La materia prima para la elaboración de este producto se adquirió en el comercio de Tacna. El procesamiento se llevó a cabo en los laboratorios de Análisis de ESIA de FCAG-UNJBG.

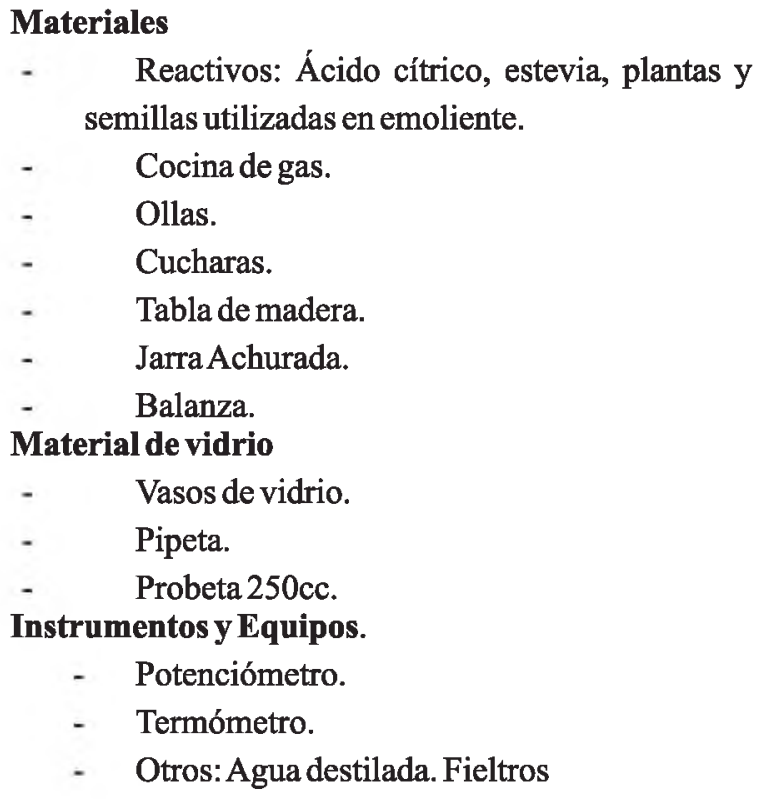

\section{Métodos}

\section{Diseño experimental}

El diagrama experimental que señala la secuencia del desarrollo de esta investigación se muestra en la figura 1 ; y el diagrama de flujo para la preparación del emoliente, en la figura 2 . Los cuales se detallan a continuación.

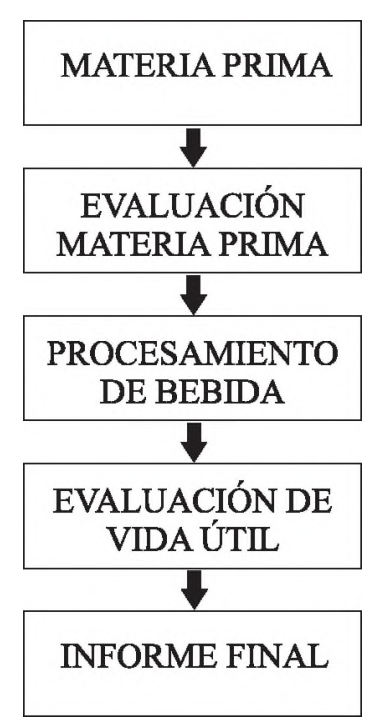

Figura 1: Diagrama experimental. Fuente: Elaboración propia. 


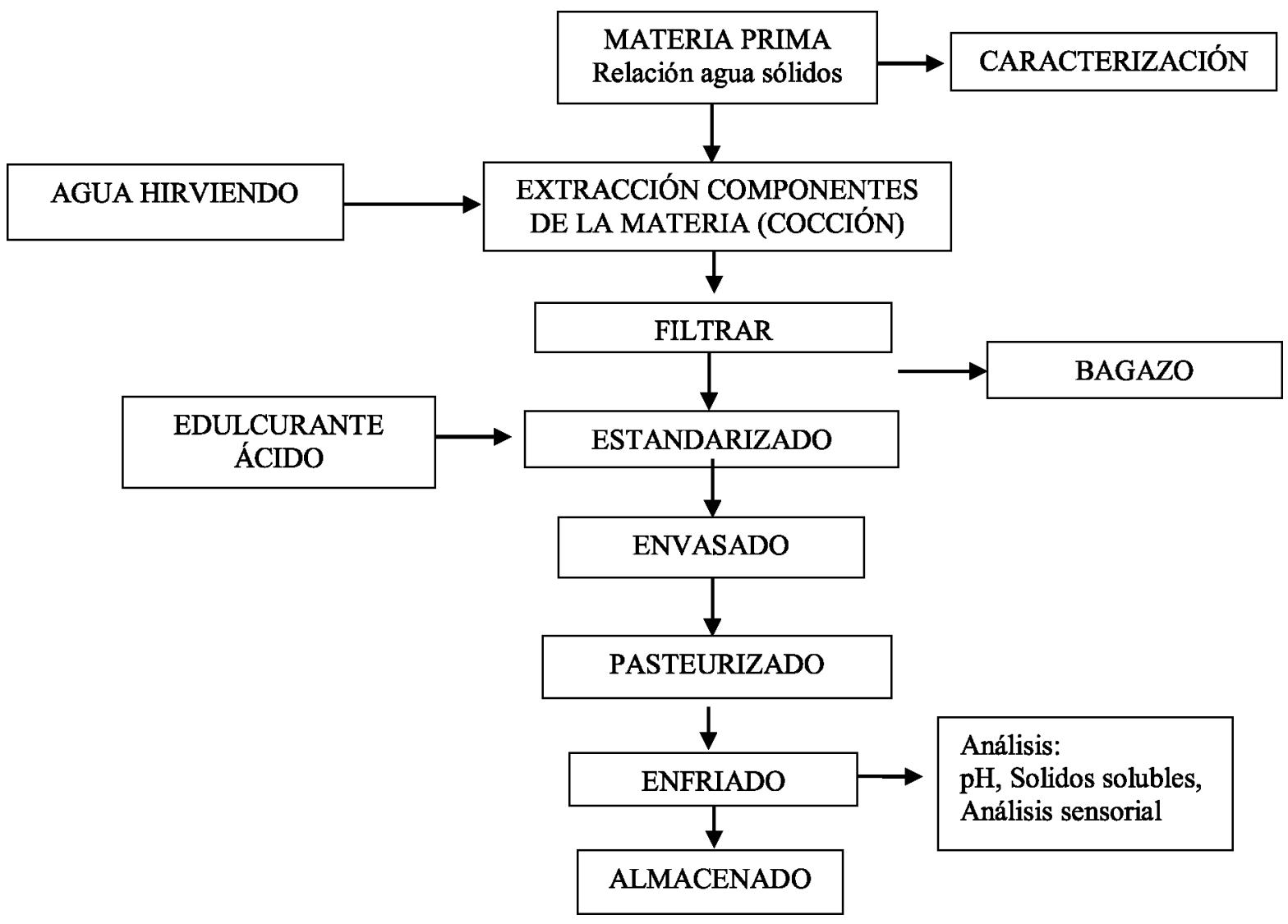

Figura 2: Diagrama de elaboración de la bebida emoliente.

Fuente: Elaboración propia.

\section{Materia prima}

Para la elaboración del emoliente Perú, se utilizaron una serie de especies vegetales que se detallarán más adelante. Estas fueron incorporadas, según la formulación comercial, en una proporción de 61 de agua potable por $178 \mathrm{gr}$ de mezcla de especies vegetales secas (emoliente).

F1 tino de esnecie vegetal seca v su narticinación norcentual en la materia nrima utilizada anarecen en la tabla 1

Tabla 1. Composición de los constituyentes de la materia prima.

\begin{tabular}{lcc}
\multicolumn{1}{c}{ NOMBRE COMÚN } & PESO (gr) & PORCENTAJE (\%) \\
\hline Cola de caballo & 6,33 & 14,9 \\
Grama & 0,17 & 0,4 \\
Boldo & 0,27 & 0,6 \\
Flor de arena & 0,45 & 1,1 \\
Uña de gato & 2,76 & 6,5 \\
Cebada tostada & 19,86 & 46,8 \\
Linaza & 42,47 & 100,00 \\
\end{tabular}

Fuente: Elaboración propia. 
Extracción de componentes de la materia prima

Con la finalidad de obtener los componentes que integran la materia prima, se realizó una extracción sólido-líquido con agua a $100^{\circ} \mathrm{C}$ por espacio de 30 minutos.

Filtrado

Se efectuó un enfriado a $85^{\circ} \mathrm{C}$, previo al filtrado para favorecer este proceso. Asimismo, se pesó el bagazo obtenido en esta etapa.

\section{Estandarizado}

Se realizó con una solución de ácido cítrico al $10 \%$ para bajar el $\mathrm{pH}$ a 3,9-4,0, y la adición de edulcorante de estevia.

Envasado

Se optó por la utilización de envases de vidrio de 450 cc de capacidad, con tapa indicadora de vacío.

Tratamiento térmico

Se aplicó el pasteurizado con temperaturas de $90^{\circ} \mathrm{C}$ por espacio de 15 minutos, luego se procedió al sellado final.

\section{Enfriado}

Se dispusieron los envases bajo un chorro suave y constante de agua potable a una temperatura de $20^{\circ} \mathrm{C}$ por un tiempo de 20 minutos.

Almacenado

Culminados los procesos anteriores, se llevó el emoliente pasteurizado a un lugar fresco, seco y con una temperatura estable de aproximadamente $22^{\circ} \mathrm{C}$.

\section{Métodos deAnálisis físico-químico}

A.-pH. (Método Potenciómetro)

B.- Sólidos solubles (Método Refractómetro).

C.- Temperatura.

E.- Panelistas: Los panelistas se seleccionaron del Curso de Control de Calidad del quinto año de la Escuela académico Profesional de Ingeniería en Industrias Alimentarias.

\section{Método estadístico para el cálculo de la vida útil}

Para esta investigación se siguieron los métodos propuestos por Espinoza (2001), los cuales se basan en los análisis sensoriales de cada panelista y la ficha de evaluación sensorial mostrada en el Anexo 1. Mediante esta evaluación sensorial, se pretende estimar la vida útil del producto con un intervalo de confianza al $95 \%$, hasta cuando el valor del sabor del emoliente sea evaluado como poco aceptable.

Para las pruebas estadísticas se ha considerado el esquema de análisis de varianza a fin de verificar si existe una relación funcional entre el tiempo de almacenamiento $(\mathrm{X})$ y el grado de aroma y sabor $(\mathrm{Y})$. El nivel de error es del $5 \%$.

a. Obtener la estimación de la vida útil para $y=3,0$
- $\quad$ Por punto

De la ecuación de regresión lineal se tiene que:

$$
\bar{x}=\frac{a-y}{b}
$$

- Por intervalo.

a) Obtener gráficamente los límites lineales superior e inferior del intervalo de confianza para la recta

Los límites deseados con un coeficiente de confianza $(1-\alpha)$ son dados por: $y=\propto+\beta x$.

$a+b x \pm d \sqrt{\text { CMerror }}\left\{\frac{1}{\sqrt{k r}}+\frac{|x-\bar{x}|}{\sqrt{r\left[\sum x^{2}-\frac{\left(\sum x\right)^{2}}{k}\right]}}\right\}$

Donde:

$\mathrm{D}=$ Valor de las tablas en función del nivel de error $\alpha$ y número de grados de libertad del error.

\section{RESULTADOS}

\section{Elaboración del emoliente}

El emoliente procesado y envasado en botellas de 300c es llevado a almacenamiento a $22^{\circ} \mathrm{C}$. En la figura 3 se muestra el producto elaborado antes de almacenarlo. Se evaluaron las características del producto elaborado, cuyas conclusiones aparecen en la tabla 2. Allí se detallan los resultados del examen sensorial que denotan la presencia elevada de la cebada tostada. En las demás características sensoriales destacan el aroma y color característicos del emoliente. Asimismo, se percibe la presencia de acidez debido a la adición de ácido cítrico para disminuir el $\mathrm{pH}$ y mejorar su conservación.

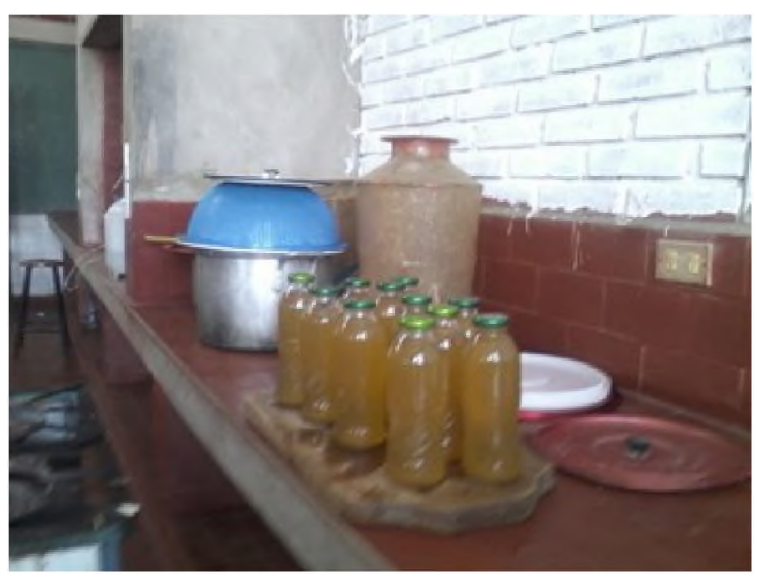

Figura 3. Emoliente envasado. Fuente: Imágenes propias. 
En la tabla 2, se resume el análisis realizado después de elaborado el producto.

Tabla 2. Resultados de análisis fisico-sensoriales realizados al producto final antes del almacenamiento.

\begin{tabular}{lll}
\hline Físicos & $\mathrm{pH}$ & 3.7 \\
\cline { 2 - 3 } & $\begin{array}{l}\text { Sólidos } \\
\text { solubles }\end{array}$ & $1,5^{\circ}$ Brix \\
\hline Sensoriales & Apariencia & Buena apariencia \\
\cline { 2 - 3 } & Color & Tono: Pardo \\
\cline { 2 - 3 } & & Intensidad: Claro \\
\cline { 2 - 3 } & Brillo: Opalescente \\
\cline { 2 - 3 } & Olor & $\begin{array}{l}\text { Característico } \\
\text { cebada tostada }\end{array}$ \\
\cline { 2 - 3 } & Textura & Buen cuerpo \\
\cline { 2 - 3 } & Sabor & $\begin{array}{l}\text { Típico predominio } \\
\text { de cebada tostada, } \\
\text { ligeramente ácido }\end{array}$ \\
\hline & $\begin{array}{l}\text { Presenta pequeño } \\
\text { sedimento }\end{array}$ \\
\cline { 2 - 3 } Fucnte: Elaboración propia. &
\end{tabular}

\section{Evaluación sensorial para determinar la vida útil del emoliente}

Se realizaron las evaluaciones sensoriales a través del tiempo. Los resultados se presentan en la tabla 3.

Tabla 3. Variación de la calidad sensorial del emoliente a través del tiempo de almacenamiento

\begin{tabular}{lllll}
\hline Fecha & \multicolumn{2}{c}{ Tiempo de almacenamiento } & \multicolumn{2}{c}{ Análisis sensorial } \\
\hline & Diferencia simple & Días (x) & Jueces & \\
& & & Yiferencia acumulada & Y1 \\
\hline $09 / 09 / 2013$ & 0 & 0 & 4,7 & 4,8 \\
$17 / 09 / 2013$ & 8 & 8 & 4,6 & 4,7 \\
$23 / 09 / 2013$ & 6 & 14 & 4,26 & 4,26 \\
$03 / 10 / 2013$ & 10 & 24 & 4,4 & 4,16 \\
$10 / 10 / 2013$ & 7 & 31 & 3,98 & 3,98 \\
$22 / 10 / 2013$ & 12 & 43 & 4,2 & 4,3 \\
$05 / 10 / 2013$ & 14 & 57 & 3,56 & 3,56 \\
$19 / 10 / 2013$ & 14 & 71 & 3,7 & 3,7 \\
$29 / 11 / 2013$ & 10 & 81 & 3,5 & 3,26 \\
$09 / 12 / 2013$ & 10 & 91 & 3,1 & 3 \\
\hline
\end{tabular}

Fuente: Elaboración propia.

Tabla 4. Análisis de Varianza

\begin{tabular}{ccccccc} 
Sesión & $\mathrm{X}$ & $\mathrm{Y} 1$ & $\mathrm{Y} 2$ & $\mathrm{Y}$ & $\mathrm{XY}$ & $\mathrm{X}^{\wedge} 2$ \\
\hline 1 & 8 & 4,7 & 4,8 & 9,5 & 76 & 64 \\
2 & 14 & 4,6 & 4,7 & 9,3 & 130,2 & 196 \\
3 & 24 & 4,26 & 4,26 & 8,52 & 204,48 & 576 \\
4 & 31 & 4,4 & 4,16 & 8,56 & 265,36 & 961 \\
5 & 43 & 3,98 & 3,98 & 7,96 & 342,28 & 1849 \\
6 & 57 & 4,2 & 4,3 & 8,5 & 484,5 & 3249 \\
7 & 71 & 3,56 & 3,56 & 7,12 & 505,52 & 5041 \\
8 & 81 & 3,7 & 3,7 & 7,4 & 599,4 & 6561 \\
9 & 91 & 3,5 & 3,26 & $\mathbf{6 , 7 6}$ & 615,16 & 8281 \\
10 & 102 & 3,1 & 3 & 6,1 & 622,2 & 10404 \\
\cline { 1 - 2 } Total & $\mathbf{5 2 2}$ & & & $\mathbf{7 9 , 7 2}$ & $\mathbf{3 8 4 5 , 1}$ & $\mathbf{3 7 1 8 2}$ \\
\cline { 1 - 4 } & & & & &
\end{tabular}

Fuente: Elaboración propia. 
Tabla 5. Análisis de Varianza.

\begin{tabular}{lcccc}
\hline \multicolumn{1}{c}{ F de V } & Gl & SC & CM & F calculado \\
\hline $\begin{array}{l}\text { Regresión } \\
\text { lineal }\end{array}$ & 1 & 5,03521224 & 5,03521224 & 648.867557 \\
Desvíos & & & & \\
regresión & 8 & 0.48966776 & 0,06120847 & 7.8876894 \\
Tiempos & 9 & 5.52488 & & \\
Error & 10 & 0.0776 & 0,00776 & \\
\hline Total & 19 & 5,60248 & & \\
\hline
\end{tabular}

Fuente: Elaboración propia.

\section{Cálculo de la vida útil del emoliente}

Análisis de varianza para verificar si existe relación funcional lineal entre el tiempo de almacenamiento $(\mathrm{X})$ y el sabor (Y)

a.- Sumas de cuadrados

Total general $=\mathrm{G}=\left(\sum \mathrm{y}\right)=79,72$

Tiempos de almacenamiento $\mathrm{k}=10$

Total de observaciones $(\mathrm{N})$

$$
N=k * r=10 * 2=20
$$

\section{Repeticiones $r=2$}

$$
\text { Media general }(\overline{\mathrm{y}})=\frac{Y}{N}=\frac{82,72}{20}=3,986 \text { Factor de corrección } C=
$$

$$
\frac{\sum Y^{2}}{N}
$$

b.- Suma de cuadrados del Total

$\mathrm{SCT}=\left(4,7^{2}+4,8^{2}+4,6+\right.$ $\left..3,0^{2}\right)-\mathrm{C}$

$\mathrm{SCT}=5,60248$

c.- Suma de cuadrados para tiempos

$\mathrm{SCt}=1 / 2 *\left(9,5^{2}+9,3^{2}+8,52^{2}+\right.$ $\left.+6.1^{2}\right)-\mathrm{C}=$

$\mathrm{St}=5,52488$

\section{SC. Regresión Lineal}

$$
S C R L=\frac{\left[\sum x y-\frac{\left.\left(\sum x\right)\left(\sum y\right)\right|_{\mid} ^{2}}{K}\right]}{r\left[\sum x^{2}-\frac{\left.\left(\sum x\right)_{1}^{2}\right]}{K}\right]_{]}}=\frac{[3845,1]-\frac{522^{*} 79,72}{10}}{2\left[37182-\frac{\left.522^{2}\right]}{10}\right]}=5,035212
$$




\section{d.- SC desvíos de la regresión lineal}

$\mathrm{SC}$ desvíos de la $\mathrm{RL}=\mathrm{SC}$ tiempos $-\mathrm{SC}$ Reg. Lineal $=0,489668$

e.- SC error

$\mathrm{SC}$ error $=\mathrm{SC}$ total-Sc tiempos $=0,0776$

$$
C V=\frac{100 \sqrt{\text { CMerror }}}{-}=\frac{100 \sqrt{0,02}}{3,986}=2,21
$$

f.- Ecuación de regresión lineal.

La ecuación de regresión lineal es expresada por:

$$
\hat{Y}=a+b * X
$$

$$
\begin{aligned}
& b=\frac{\left[\sum x y-\frac{\left.\left(\sum x\right)\left(\sum y\right)_{!}\right]}{K}\right]}{r\left[\sum x^{2}-\frac{\left.\left(\sum x\right)_{1}^{2}\right]}{\left.K\right|_{]}}\right.}=\frac{[4621,38]-\frac{522 * 82,72}{10}}{2\left[37182-\frac{\left.522^{2}\right]}{10}\right]}=-0,01592 \\
& \begin{array}{c}
a=\bar{y}-b * \bar{x}=4,817019 \\
\hat{Y}=4,817019-0,01592 * X
\end{array}
\end{aligned}
$$

\section{Calculando la vida útil}

a.-Aplicando en la ecuación lineal la calidad límite. $\mathrm{Y}=3$ (Valor límite del análisis sensorial)

$$
X=\frac{4,817019-\bar{y}}{0,01592}=114,135 \text { días }
$$

Para un $\alpha=0,05$ se estimó la vida útil del emoliente procesado en 114 días.

Para un $\alpha=0,05$ se estimó la vida útil del emoliente procesado en 114 días.

\section{DISCUSIÓN DE RESULTADOS}

El emoliente es una bebida que se prepara sobre la base de granos tostados de cebada, extractos de hierbas medicinales, azúcar y jugo de limón. Entre las hierbas más usadas se encuentran la cola de caballo, la linaza, la alfalfa, el llantén y el boldo. Estos sufren ligeras variaciones debido al tipo de cultivo que realiza el productory a los fines medicinales que se buscan.

$$
\mathrm{R}^{2}=0,91
$$

El emoliente obtenido presentó bajo nivel de sólidos solubles gracias al uso de estevia en lugar de sacarosa. A ello se sumó la ventaja adicional de la estevia que la vuelve no fermentescible por los hongos y levaduras.

$\mathrm{El} \mathrm{pH}$ final de emoliente permite un tratamiento térmico de pasteurización.

Asimismo, se pudo observar como parte de los atributos sensoriales del emoliente, una buena apariencia; sobresalió el olor y sabor del componente de la materia prima cebada; y además presentó un pequeño sedimento.

\section{Vida útil del emoliente}

Se efectuaron pruebas sensoriales iniciales a la preparación del emoliente y se encontró una valoración no muy alta en los resultados promedios; los que se pueden señalar como aceptables, inclusive al inicio son un tanto más bajos. Dichos valores persisten a través de los 102 días que duró la evaluación sensorial; al término de este periodo, se notó un incremento de sedimento en la base de la botella, generado por los componentes sólidos de la preparación; los cuales ya daban muestras de cierto aspecto turbio al inicio del proceso de vida útil de la bebida. (Estabilidad física). 
Se debe señalar que la alteración microbiológica esperada en este tipo de producto se debe básicamente a la interacción de levaduras y hongos, por las características de su composición (sabor alcohólico), la cual no se presentó en ningún momento; fue una degradación interna de los componentes la que afectó el sabor volviéndolo muy ligero e incrementando un tanto el amargor. Por ello fue disminuyendo su valoración a cargo de los panelistas, quienes determinaron el final de prueba por tener un valor de 3 en la escala sensorial utilizada; interpretándose como poco aceptable.

\section{Aplicación del método regresión lineal para predecir la vida útil del emoliente}

A partir de estos datos experimentales, se utilizó la regresión lineal para estimar la vida útil del emoliente y se halló la ecuación que modela la pérdida de calidad estableciéndose un valor de 3 de la escala sensorial, sometiéndola al modelo y encontrándose un valor de 15 meses como fecha límite para su consumo. Esto se consideró como un incremento importante de la vida útil del producto que permitirá una mejor comercialización y un paso para su industrialización y exportación.

Cabe señalar que esta predicción de vida útil es a $22^{\circ} \mathrm{C}$.

\section{CONCLUSIONES}

El tiempo de vida útil del emoliente peruano elaborado, aplicando el tratamiento térmico y envasándolo en botellas de vidrio, incrementa significativamente su perdurabilidad de 2 a 114 días, a una temperatura de almacenamiento de $22^{\circ} \mathrm{C}$.

\section{REFERENCIAS BIBLIOGRÁFICAS}

Acosta, M. (2011). La historia del Emoliente en Diario "El Comercio" 21 de mayo de 2011. $\mathrm{R}$ e c u p e r a d o d e : https://elcomercio.pe/gastronomia/.../ historia-emoliente-bebida-esquinanoticia-76046

Andalúa, M. (1994). Evaluación Sensorial de Alimentos en la teoría y en la Práctica. Editorial Acribia. Zaragoza España.

Biblioteca Central de la Universidad Nacional de Santa (2011) Vida útil de alimentos. Recuperado de: Biblioteca.uns.edu.pe $\begin{array}{lllll}/ & \mathbf{s} & \mathbf{a} & 1 & \mathrm{a}\end{array}$ docente/archivos/cursos/aula_ 2 iii..

Charm, S. (2007). Food engineering applied to accommodate food regulations, quality and testing. Alimentos Ciencia $e$ Ingeniería. 16(1), 5-8.

Charley. Cit (1987). Tecnología de Alimentos.
Procesos químicos y físicos. Editorial Limusa. México.

Chourrout Virginia Evaluación sensorial: Estudio de la vida útil de alimentos y bebidas (2011). Recuperado de: http://www.alimentacion.enfasis.com/ articulos/18043-Eevaluacionsensorial-estudio-la-vida-utilalimentos-v-bebidas

Ecoticias (2015). Los dulces beneficios de la stevia y sus propiedades. Recuperado de: https://www.ecoticias.com/naturaleza/ 27352/Los-dulces-beneficios-de-laStevia-v-sus-propiedades.

EL Cocinero Peruano (2018) El Emoliente. $\mathrm{R}$ e c u p e r a d o d e : http://www.cocineroperuano.com/beb idas/160-el-emoliente.html

Emoliende Etmobotanico (2010). Recuperado de: http://emolientetnobotanico.blogspot. com/

Origen del Emoliente peruano (2008). Recuperado de: http://elperuanisimoemoliente.blogsp ot.com/2008/1

Espinoza, E. (2001). Evaluación Sensorial de Alimentos. Tacna, Perú.

Espinoza, E. (2003). Diseño Experimental en la Evaluación Sensorial de Alimentos. Tacna, Perú.

Montes M (2012). Bebida Natural y curativa busca conquistar conquistar América. $\mathrm{R}$ e c u p e r a d o d e : http://www.eluniversal.com.co/cartag ena/actualidad/bebida-natural-vcurativa-busca-conquistar-america$\underline{72147}$

Posada Carla. (2011) Recopilación de estudios de vida út $i l$. $\mathrm{R} \mathrm{e} \mathrm{c} \mathrm{u} \mathrm{p} \mathrm{e} \mathrm{r}$ a d o $\mathrm{de}$ : http://repositorv.lasallista.edu.co/dspa ce/bitstream/10567/683/1/Recopilacio $\underline{n}$ estudios vida util.pdf.pdf

Pedrero D \& Pangborn R (1989). Evaluación sensorial de Alimentos. Métodos Estadísticos. Editorial Alhambra. Madrid, España.

Varman, A y Sutherland. (1994). Bebidas. Editorial Acribia-España.

Vida Útil de Alimentos. (2011) Recuperado de: biblioteca.uns.edu.pe/sala docente/archivos/cursos/aula_2_iii...R ecuperado en junio 2013.

Vida OK (2013). Benéficos de los Emolientes preparados con hiervas medicinales. R e c u p e r a d o d e : https://vidaok.com/emolientebeneficios-hierbas-medicinales/- 